\title{
Analysis of Blind Separation of Noisy Mixed Images Based on Wavelet Thresholding and Independent Component Analysis
}

\author{
Alka Mahajan, Member, IEEE, and Gajanan Birajdar
}

\begin{abstract}
This paper investigates the technique of wavelet threshold de-noising with Independent Component Analysis (ICA) for noisy image separation. In the first approach, noisy mixed images are separated using fast ICA algorithm and then wavelet thresholding is used to de-noise. The second approach uses wavelet threshold to de-noise and then use the fast ICA algorithm to separate the de-noised images. The simulation results show better performance of image separation followed by denoising rather than the other way round. Peak Signal to Noise Ratio (PSNR), Improved Signal to Noise Ratio (ISNR), Signal to Noise Ratio (SNR) and Root Mean Square Error (RMSE) are used to evaluate quality of separated images. Amari error and structural similarity index (SSIM) is computed for the separation quality measurement.
\end{abstract}

Index Terms-Blind source separation (BSS), fast ICA, Independent component analysis (ICA), Wavelet threshold.

\section{INTRODUCTION}

Digital images are invariably contaminated by noise. Noise arises due to imperfect instruments used in image processing, problems with the data acquisition process, and interference which can degrade the data of interest. Also, noise can be introduced due to compression and transmission errors [1]. The overall noise characteristics in an image depend on factors like, type of sensor, exposure time, pixel dimensions, ISO speed, and temperature [2]. As most of the natural images are assumed to have additive random noise, which is modeled as Gaussian type, denoising is the first step to be considered before the image data is analyzed.

Blind source separation (BSS) is the method of extracting underlying source signals from a set of observed signal mixtures with little or no information as to the nature of these source signals. Independent component analysis (ICA) is used for finding factors or components from multivariate statistical data and is one of the many solutions to the BSS problem [3]-[5]. ICA looks for the components that are both statistically independent and nongaussian. The various ICA algorithms extract source signals based on the principle of information maximization, mutual information minimization, maximum likelihood estimation and maximizing nongaussianity. ICA is widely used in statistical signal processing, medical image processing, economic analysis and telecommunication applications [6]-[7].

Most of the ICA methods are developed assuming

Manuscript received July 11, 2011, revised September 22, 2011.

Alka Mahajan and Gajanan Birajdar are with S.I.E.S. Graduate School of Technology, Navi Mumbai, India. (e-mail: alkamahajan@gmail.com; gajanan123@gmail.com). noiseless data and these algorithms perform poorly in the presence of noise [14]. In this paper, noisy multiple channel blind signal separation algorithms based on wavelet thresholding are investigated. In the first approach, noisy mixed images are separated using fast ICA algorithm and then soft wavelet thresholding is used to de-noise. Second approach uses soft wavelet thresholding to de-noise and then the use of the fast ICA algorithm to separate the de-noised images.

\section{PRINCIPLE OF IndEPENDENT COMPONENT ANALYsis}

Many popular ICA methods use a nonlinear contrast function to blindly separate the signals. Examples include equivariant adaptive source separation [8], fast ICA [9], and efficient Fast ICA [10]. Adaptive choices of the contrast functions have also been proposed, in which the probability distributions are obtained by considering a maximum likelihood (ML) solution corresponding to some given distributions of the sources and relaxing this assumption afterward [11]-[12]. This method is specially adapted to temporally independent non-Gaussian sources and is based on the use of nonlinear separating functions. Further Tichavsky et al. [13] have proposed two general purpose rational nonlinearities that have similar performance as tanh, but can be evaluated faster.

The basic ICA model which is shown in Fig. 1can be stated as,

$$
x(t)=A s(t)+n(t)
$$

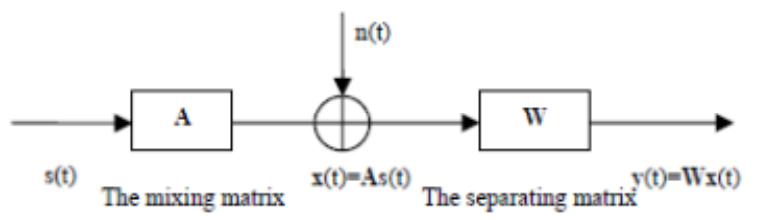

Fig. 1. Noisy ICA model

where $x(t)$ is a $N$ dimensional vector of observed signals at the discrete time instant $t$, A is an unknown mixing matrix, $s(t)$ is original source signal of $M \times N(M \leq N)$ and $n(t)$ is the observed noise vector and $\mathrm{M}$ is number of sources.. The purpose of ICA is to estimate $s(t)$ which is the original source signal, from $x(t)$ which is the mixed signal, i. e. it is equivalent to estimating matrix $A$. Assuming that there is a matrix $W$, which is the de-mixing matrix or separation inverse matrix of $A$, then the original source signal is obtained by

$$
s(t)=W x(t)
$$


ICA algorithm assumes [14] that the mixing matrix $A$ must be of full column rank and all the independent components $s(t)$, with the possible exception of one component, must be non-Gaussian. Further, the number of observed linear mixtures $m$ must be at least as large as the number of independent components $n(m \geq n)$.

\section{Denoising Methods}

Image noise removal is traditionally achieved by time domain and frequency domain processing techniques. The classical method in time domain processing techniques is wiener filtering [15] which is optimal in the least MSE sense while wavelet threshold denoising [16] is a frequency domain processing technique that is based on the notion of optimal time-frequency localization. The Wavelet analysis has a resolution in time and frequency domain. Using multi-scale analysis, the detail local features can be revealed under various scales. In wavelet thresholding, a signal is decomposed into low-frequency and high-frequency sub-bands. The high-frequency sub-band coefficients are processed using hard or soft thresholding. As compared to wiener filtering, wavelet thresholding performance is better [17]. Wavelet thresholding denoising method is explained in the following section.

\section{A. Wavelet Threshold Approach}

The theory of wavelet threshold de-noising is based on multi-resolution analysis of Wavelet transform [18] - [21]. After multi-resolution analysis, the data is divided into an approximation sequence and a detail sequence at each resolution. In wavelet thresholding, each coefficient is thresholded (set to zero) by comparing against a threshold to eliminate noise, while preserving important information of the original signal. Usually two types of thresholding techniques are used: the soft threshold and hard threshold. 1) Hard Threshold:

The hard thresholding operator is defined as

$$
y=\left\{\begin{array}{cc}
x & |x|>T \\
0 & \text { otherwise }
\end{array}\right.
$$

where $T=$ given threshold value and the original signal is expressed as $y=x$.

\section{2) Soft Threshold:}

The soft thresholding operator is defined as

$$
y=\left\{\begin{array}{rr}
\operatorname{sign}(x) \cdot(|x|-T) & |x|>T \\
0 & \text { otherwise }
\end{array}\right.
$$

As soft threshold has better mathematical characteristics, soft threshold method is used for denoising. Soft thresholding shrinks the magnitudes of the coefficients above the threshold in absolute value and this method is used as the thresholding technique in this paper. Determination of the value of the threshold is crucial as larger value may result into loss of information while smaller one may allow noise to continue.

\section{FASTICA ALGORITHM}

Fast ICA is the most popular algorithm used in various applications as it is simple, fast convergent and computationally less complex. It is a fixed point iteration scheme for finding a maximum of non-gaussianity or negentropy of $W^{T} x$ i.e. one of the independent component $\left(S_{i}=W_{i}^{T} x\right)$. Fast ICA learning rule finds the direction of a unit vector ' $w$ ' such that the projection of $W^{T} x$ maximizes nongaussianity. Nongaussianity is measured by negentropy $J\left(W^{T} x\right)$ i.e.

$$
J(y)=[E\{G(y)\}-E\{G(V)\}]^{2}
$$

where $v$ is Gaussian variable of zero mean and unit variance and $G$ is some non-quadratic function. The variable $y$ is assumed to be zero mean and unit variance. The fastICA algorithm was applied to a set of natural images.

Data pertaining to the selected natural images was first subjected to preprocessing before applying to ICA algorithm. Preprocessing is necessary to simplify and reduce the complexity of the problem.

The steps applied in the fastICA are as listed below: 1. The data $x$ is centered by subtracting its mean vector $m=E\{x\}$ so as to make $\mathrm{x}$ a zero mean variable.

2. The observed data $x$ is transformed linearly so that a components of a new vector $\tilde{x}$ are uncorrelated and their variance equals unity i.e. $E\left\{\tilde{x}^{T}\right\}=I$. This whitening is done by using eigenvalue decomposition (EVD) of the covariance matrix.

The new mixing matrix obtained using whitening is given by,

$$
\tilde{x}=E D^{-1 / 2} E^{T} x=E D^{-1 / 2} E^{T} A s=\tilde{A} x
$$

3. An initial random mixing matrix $w$ is chosen.

4. Let,

$$
w^{+}=E\left\{x g\left(w^{T} x\right)\right\}-E\left\{g^{\prime}\left(w^{T} x\right)\right\} w
$$

where $g$ is the nonlinearity function.

5. The matrix $w$ is then orthogonalized.

6. Let,

$$
w=\frac{w^{+}}{\left\|w^{+}\right\|}
$$

7. Step 4 is repeated till convergence is obtained. Convergence means that the old and new values of $w$ point in the same direction i.e. their dot product equal to 1 . To estimate several independent components, we need to run one unit FastICA algorithm using several units with weight vectors $w_{1}, \ldots, w n$. When we have estimated $p$ independent components, or $p$ vectors $w_{l}, \ldots, w_{p}$ we run the one unit fixed point algorithm for $w_{p+l}$, and after every iteration step subtract from $w_{p+1}$ the projections $w_{p+1}^{T} w_{j} w_{j}, j=1, \ldots, p$ of the previously estimated $\mathrm{p}$ vectors, and then renormalize $\mathrm{w}_{\mathrm{p}+1}$

Let

$$
\begin{aligned}
& w_{p+1}=w_{p+1}-\sum_{j=1}^{p} w_{p+1}^{T} w_{j} w_{j} \\
& w_{p+1}=w_{p+1} / \sqrt{w_{p+1}^{T} w_{p+1}}
\end{aligned}
$$

The various performance factors are then calculated using the following equations.

(i) Root mean square error (RMSE):

$$
\mathrm{RMSE}=\sqrt{\frac{1}{\mathrm{MN}} \sum_{\mathrm{i}=1}^{\mathrm{N}} \sum_{\mathrm{j}=1}^{\mathrm{M}}\left(\mathrm{I}_{\mathrm{ij}}-\tilde{\mathrm{I}}_{\mathrm{ij}}\right)^{2}}
$$


where $N \times M=$ Image size, $I i j=$ original source image,

$\tilde{I}_{i j}=$ separated image

(ii) Peak signal to noise ratio $(P S N R)$ :

$$
\mathrm{PSNR}=20 \log _{10}\left(\frac{255}{\mathrm{RMSE}}\right) \mathrm{dB}
$$

(iii) Signal to noise ratio (SNR):

$$
\mathrm{SNR}=10 \log _{10} \frac{\sum_{\mathrm{x}=0}^{\mathrm{M}-1} \sum_{\mathrm{y}=0}^{\mathrm{N}-1} \mathrm{f}(\mathrm{x}, \mathrm{y})^{2}}{\sum_{\mathrm{x}=0}^{\mathrm{M}-1} \sum_{\mathrm{y}=0}^{\mathrm{N}-1}[\mathrm{f}(\mathrm{x}, \mathrm{y})-\tilde{\mathrm{f}}(\mathrm{x}, \mathrm{y})]^{2}} \quad \mathrm{~dB}
$$

where $f(x, y)=$ original image and $f(x, y)=$ separated image (iv) Improved signal to noise ratio (ISNR):

$$
I S N R=10 \log _{10}\left[\frac{s(m, n)-x(m, n)}{s(m, n)-y(m, n)}\right]
$$

where $s(m, n)=$ source image, $x(m, n)=$ mixed image, $y(m, n)=$ separated image

(v) Amari error is then used for evaluating separation quality of unmixing matrix $w$ and to compare various ICA algorithms [7]. Before calculating Amari error, normalization is carried out on the mixing matrix and the estimated separation matrix. This normalization is done in the range of $[0,1]$ where 0 corresponds to perfect separation. It is given by the equation,

$$
d(W, A)=\frac{1}{2 m(m-1)} \times\left\{\sum_{i=1}^{m}\left[\frac{\sum_{j=1}^{m}\left|a_{i j}\right|}{\max _{j}\left|a_{i j}\right|}-1\right]+\sum_{j=1}^{m}\left[\frac{\sum_{i=1}^{m}\left|a_{i j}\right|}{\max _{i}\left|a_{i j}\right|}-1\right]\right\}
$$

where, $a_{i j}=\left(W^{l} A\right)_{i j}$, and $W$ and $A$ are $n \times m$ matrices.

(vi) Mean Structural similarity index (MSSIM):

The SSIM metric is calculated on various windows of an image. The measure between two windows $x$ and $y$ of common size $N \times N$ is [22]:

$$
\operatorname{MSSIM}(X, Y)=\frac{1}{M} \sum_{j=1}^{M} \operatorname{SSIM}\left(x_{j}, y_{j}\right)
$$

where $X$ and $Y$ are the reference and the distorted images, respectively; $x_{j}$ and $y_{j}$ are the image contents at the $j$ th local window; and $M$ is the number of windows of the image.

$$
\operatorname{SSIM}(\mathrm{x}, \mathrm{y})=\frac{\left(2 \mu_{\mathrm{x}} \mu_{\mathrm{y}}+\mathrm{C}_{1}\right)\left(2 \sigma_{\mathrm{xy}}+\mathrm{C}_{2}\right)}{\left(\mu_{\mathrm{x}}^{2}+\mu_{\mathrm{y}}^{2}+\mathrm{C}_{1}\right)\left(\sigma_{\mathrm{x}}^{2}+\sigma_{\mathrm{y}}^{2}+\mathrm{C}_{2}\right)}
$$

With $\sigma_{\mathrm{x}}$ is the mean intensity of $\mathrm{x}, \sigma_{\mathrm{y}}$ is the mean intensity of $y, \sigma_{x}^{2}$ is the variance of $x, \sigma_{y}^{2}$ is the variance of $y, \sigma_{x y}$ is the covariance of $\mathrm{x}$ and $\mathrm{y}, C_{1}=\left(k_{1} L\right)^{2}, C_{2}=\left(k_{2} L\right)^{2}$ two variables to stabilize the division with weak denominator; $\mathrm{L}=$ where is the dynamic range of the pixel values ( 255 for 8 -bit grayscale image), $k_{1}=0.01$ and $k_{2}=0.03$ by default.

\section{Simulation Results}

In this study, two images [23] Airplane and Chemical plant of size $256 \times 256$ were selected which are shown in Fig. 2 .

White Gaussian noise was added in these images which are shown in Fig. 3. These noisy images were mixed using $2 \times 2$ random mixing matrix. The mixed images so obtained are shown in Fig. 4. Simulations were performed using Matlab ${ }^{\circledR}$ R 7.9 on a Pentium IV 1.6 GHz PC. In first approach, noisy mixed images were separated by applying fast ICA algorithm first and then denoising the separated independent components was done using Soft wavelet thresholding. Images which are separated by this approach are shown in Fig. 5. Second approach uses wavelet thresholding to de-noise and then fast ICA algorithm is used to separate the de-noised images. The output images of this approach are shown in Fig. 6. Output images are scaled between [0, 255]. Performance parameters obtained using the wavelet thresholding approach are tabulated in table I and II. Similarly, Amari error was calculated and it was found that the Amari error was 0.27 with the first approach and 0.3 with the second approach indicating better performance with the former. Image separation quality was obtained by evaluating RMSE, PSNR, SNR, ISNR and SSIM.
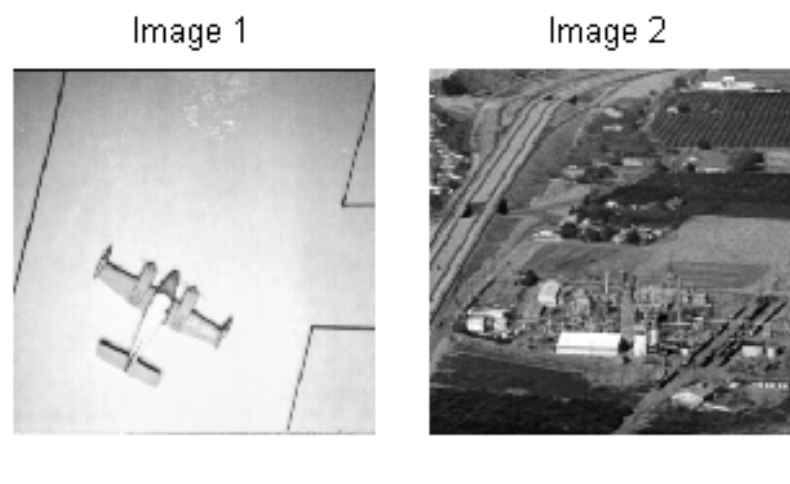

Fig. 2. Original Images

\section{Noisy Image 1}

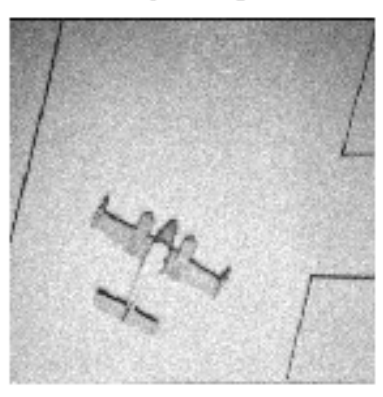

Fig. 3. Noisy images

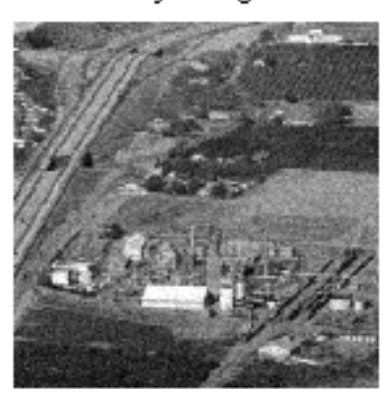

Noisy Image 2

Mixed Image 1

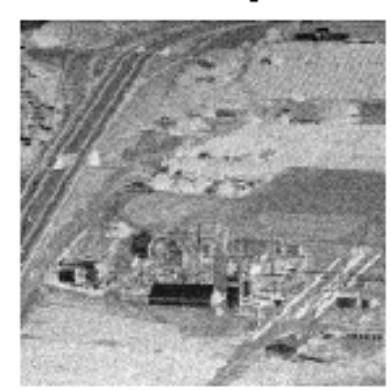

Mixed Image 2

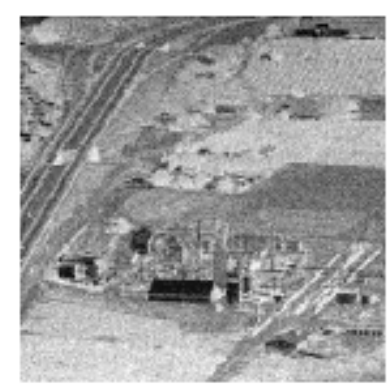

Fig. 4. Noisy mixed images 
Wavelet Filtered Image 1
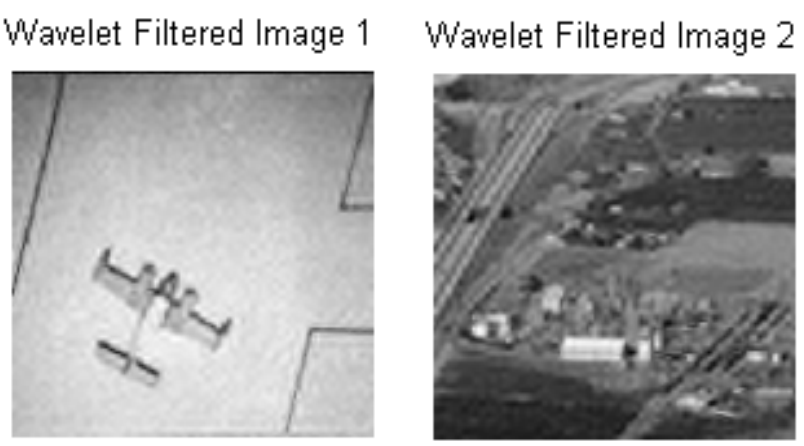

Fig.5. Approach 1 - unmixing first and then denoising output
Image 1

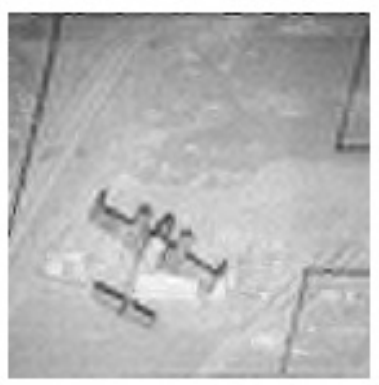

Image 2

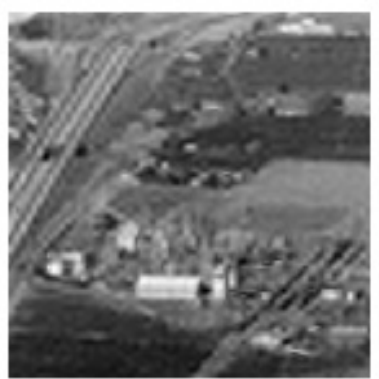

Fig.6. Approach 2 - denoising first and then unmixing - output

It is observed from Table I - II that, the blind source separation using the first approach i.e. un-mixing image first and then denoising results in $20-25 \%$ improvement of SNR, PSNR, RMSE, SSIM and ISNR as compared to denoising first and then un-mixing thereby resulting in better source separation with minimum distortion.

TABLE I: APPROACH 1: UN-MIXING IMAGE FIRST AND THEN DENOISING

\begin{tabular}{ccc}
\hline \hline Parameter & Image 1: Airplane & Image 2: Chemical Plant \\
\hline SNR & 25.6563 & 18.0047 \\
ISNR & 28.8988 & 21.9371 \\
PSNR & 27.9261 & 25.0947 \\
RMSE & 10.0947 & 14.1842 \\
MSSIM & 0.8553 & 0.6755 \\
\hline \hline
\end{tabular}

TABLE II: APPROACH 2: DENOISING FIRST AND THEN UN-MIXING

\begin{tabular}{ccc}
\hline \hline Parameter & Image 1: Airplane & Image 2: Chemical Plant \\
\hline SNR & 20.5400 & 5.9019 \\
ISNR & 23.7825 & 9.8345 \\
PSNR & 22.8098 & 12.9919 \\
RMSE & 18.4523 & 57.1409 \\
MSSIM & 0.7613 & 0.3619 \\
\hline \hline
\end{tabular}

\section{CONCLUSION}

In this paper two approaches of noisy mixed image separation have been studied to observe the effect of denoising before and after image separation. Denoising was done using soft wavelet thresholding considering its better performance in terms of PSNR \& RMSE over hard thresholding. It was observed that the first approach of un-mixing image first and then denoising results in better source separation with minimum distortion in terms of SNR, PSNR, RMSE, SSIM and ISNR as compared to denoising first and then un-mixing.

\section{REFERENCES}

[1] M. Motwani, M. Gadiya, R. Motwani, F. Harris, Jr, "Survey of Image Denoising Techniques," Proc. of GSPx 2004, Santa Clara Convention Center, Santa Clara, CA, 2004, pp. 27-30,

[2] Ming Zhang and Bahadir Gunturk, "A New Image Denoising Method based on the Bilateral Filter", ICASSP IEEE, pp. 2008, 929-932.

[3] A. Hyvärinen and Erkki Oja, "A fast fixed-point algorithm for independent component analysis", Neural Computation, 9(7):1483-1492, 1997.

[4] Bell, A. J. and Sejnowski, T. J, "An information-maximization approach to blind separation and blind deconvolution", Neural Computation, 7(6):1129-59, 1995.

[5] J. F. Cardoso, and A. Souloumiac, "Blind beamforming for non-Gaussian signals", IEE Proceeding Part F, Vol.140, No. 6: 362-370, 1993.

[6] A. Hyvärinen, J. Karhunen, E. Oja, Independent Component Analysis, John Wiley \& Sons, , 2001.

[7] Aapo Hyvärinen and Erkki Oja, "Independent Component Analysis: Algorithms and Applications", Neural Networks, 13(4-5):411-430, 2000.

[8] Cardoso J.F., Laheld B., "Equivariant adaptive source separation" IEEE transaction Signal Processing, 45, pp. 434-444, 1996.

[9] A. Hyvärinen and Erkki Oja, "A fast fixed-point algorithm for independent component analysis", Neural Computation, 9(7):1483-1492, 1997.

[10] Koldovsky Z., Tichavsky P., Oja E., "Efficient variant of algorithm fastICA for independent component analysis attaining the Cramer-Rao lower bound" IEEE transactions Neural Networks, 17, pp. 1265-1277, 2006.

[11] Karvanen, J., Eriksson, J., Koivunen, V., "Maximum likelihood estimation of ICA model for wide class of source distributions", Neural Networks for Signal Processing 1, pp. 445-454, 2000.

[12] Pham, D.T., Garat, P., "Blind separation of mixture of independent sources through a quasi-maximum likelihood approach", IEEE Transaction Signal Processing, 45, pp. 1712- 1725, 1997.

[13] P. Tichavsky, Z. Koldovsky and E. Oja, Sept. "Speed and Accuracy Enhancement of Linear ICA Techniques Using Rational Nonlinear Functions", Proceedings of 7th International Conference on Independent Component Analysis (ICA2007), pp. 285-292, 2007.

[14] A. Hyvärinen, "Survey on Independent Component Analysis", Neural Computing Surveys, 2, 94-128, 1999.

[15] F.Lei, F.Iton, T.Yatagai, "Adaptive binary transform correlator for image recognition”, Applied Optics, 41 (35), pp. 416-7421, 2002.

[16] Tai-Chiu Hsung, "Optimizing the multiwavelet shrinkage denoising”, IEEE Trans on Signal Processing, 53 (1), pp. 240-250, 2005.

[17] P. Mayo, F. Rodenas, G. Verdú, "Comparing Methods to Denoise Mammographic Images", Proceedings of the 26th Annual International Conference of the IEEE EMBS San Francisco, CA, USA, September 1-5, 2004, pp. 247-250.

[18] Hao Zhao, Weidong Zhou, Yuhua Peng, “Application of Independent Component Analysis on Noisy Image Separation", 7th International Conference on Signal Processing, ICSP '04, Volume: 2, pp: 1018 $1021,2004$.

[19] Hong-yan Li, Qing-hua Zhao, Jing-qing Zhao, Bao-jin Xiao, "Blind Separation of Noisy Mixed Images Based on Wiener Filtering and Independent Component Analysis", 2nd International Congress on Image and Signal Processing, CISP '09, pp. 1-5, 2009.

[20] P. Mayo, F. Rodenas, G. Verdú, "Comparing Methods to Denoise Mammographic Images", Proceedings of the 26th Annual International Conference of the IEEE EMBS, pp. 247-250, 2004.

[21] Zheng-Hong Huang, "Image denosing by Independent Component Analysis based on dynamic wavelet transform", International Conference on Wavelet Analysis and Pattern Recognition ICAPR'09, pp. 351-354, 2009.

[22] Zhou Wang, Alan Conrad Bovik, Hamid Rahim Sheikh, Eero P. Simoncelli, "Image Quality Assessment: From Error Visibility to Structural Similarity", IEEE TRANSACTIONS ON IMAGE PROCESSING, VOL. 13, NO. 4, pp.600-612, 2004.

[23] Image Database URL: http://sipi.usc.edu/database/index.php 
Dr. Alka Mahajan received B.E ((Electrical and Electronics) from Andhra University, Waltair (A. P.) in 1983, M.Tech (Electronics Design and technology) from CEDTI Aurangabad India in 1994 and Ph.D. (Electronics and communication) from Delhi university, New Delhi, India in 2007. She has over 25 years of teaching and research experience. Currently, she is Principal and Professor of Electronics in S.I.E.S. graduate School of Technology, Navi Mumbai, India.

She is member of IEEE, IETE, IEI, ISTE and CSI. Her research interest are in Digital Signal and Image Processing, Soft Computing and Artificial Intelligence.

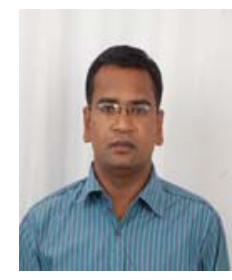

Gajanan Birajdar is working as Assistant Professor in the department of Electronics \& Telecommunication Engineering at SIES Graduate School of Technology, Navi Mumbai, India. He obtained B.E. (Electronics) from Dr. BAM University in 1996, Aurangabad, Maharashtra and M.

Tech. (Elect. \& Telecom) from Dr. BAM Technological University, Lonere, India in 2004. He has been in teaching for the past 14 years. He is life member of ISTE and IETE. He has published papers in international journals. His area of research includes Image and Speech Processing. 